\title{
Identification and diagnosis of high-precision positioning systems using the principal components method
}

\author{
Anton M. Lankin*, Mariya Y. Lankina, and Mikhail $V$. Lankin \\ Platov South-Russian State Polytechnic University, 346400 Novocherkassk, Russia
}

\begin{abstract}
The aim of this paper is to develop the theoretical basis of highprecision systems construction using principal components projection method for conducting natural model measurements of high-precision systems parameters. It is difficult to name a technical sphere, where highprecision systems systems aren't applied. They can be found in many household appliances, communication devices, they are an integral part of electrical machinery, many devices of industrial automation, control equipment and protection of various electrical installations. To conduct natural model measurements we need models connecting various parameters of high-precision systems systems between each other.
\end{abstract}

\section{Introduction}

To carry out a model simulation experiment, models that relate the parameters of highprecision systems of different physical nature [1-6]. A model-natural experiment allows to achieve the coincidence of the greatest number of the model and the object parameters [7, 8]. It combines the measurement of a physical object and the modeling of the processes taking place in this object with the means of a personal computer. In our case, the highprecision system is the physical object for carrying out the model simulation experiment. High-precision systems are the combination of precision mechanics and modern electric, electronic and computer control elements. Thus, in case of a natural-model experiment, it is required to construct multi-physical models linking the electrical, magnetic, and mechanical processes that occur in them. To consider the reliability of high-precision systems thermal processes must to be taken into account. Thus multi-physical model of high-precision systems is a system of equations:

$$
\begin{cases}P \text { elec }=f 1(U, I, R, \psi), & \text { electrical processes } \\ P \text { mag }=f 2(\psi, B(H), I, G), & \text { magnetic processes } \\ P \text { mech }=f 3(m, x, G, I), & \text { mechanical processes } \\ P \text { ther }=f 4(T, Q, I, G), & \text { thermal processes }\end{cases}
$$

\footnotetext{
* Corresponding author: semenchenko-mariya@mail.ru
} 
where Pelec, Pmag, Pmech, Pther - dependent model parameters, $f$ - independent parameters of the model: $U$-voltage, $I$ - current, $R$ - resistance, $\psi$-magnetic flux, $B(H)-$ magnetic characteristics, $m$ - weight, $x$ - gap, $G$ - generalized dimensional figure, $T-$ temperature, $Q$ - thermal energy.

The type and parameters of such multi-physical model can be found basing on the description of physical processes of interaction and can be determined by statistical methods using the results of a number of measurements.

\section{Methods and researches}

The type and parameters of such multi-physical model can be found basing on the description of physical processes of interaction and can be determined by statistical methods using the results of a number of measurements.

Second method model development is using matrix independent $\mathbf{X}$ and dependent $\mathbf{Y}$ variables. Connection between $\mathbf{X}$ and $\mathbf{Y}$ can be set using multiple regression [9].

In matrix form, the formula of model development could be seen as

$$
\mathbf{Y}=\mathbf{A} \mathbf{X}
$$

where $\mathbf{A}$-matrix of the model parameters, connecting matrix of independent $\mathbf{X}$ and dependent $\mathbf{Y}$ variables.

To determine the matrix parameters of model $\mathbf{A}$ the following expression could be used:

$$
\mathbf{A}=\mathbf{X}^{-1} \mathbf{Y} \text {. }
$$

However, it is worth considering the fact that by making such multi-physical modelling the problem of source data multicollinearity appeares. Multicollinearity of initial data implies a high degree of correlation between the elements of the matrices $\mathbf{X}$ and $\mathbf{Y}$. In this case the application of multivariate regression methods is unacceptable, as the obtained results are ambiguous and inadequate.

One of the effective ways to cope with multicollinearity is the use of projection methods, which are based on principal component analysis (PCA) [10, 11]. PCA allows converting data into a new orthogonal basis, where there will be no phenomenon of source data multicollinearity [12].

Let's consider the case when both matrices $\mathbf{X}$ and $\mathbf{Y}$ show multicollinearity. In this case the transition from an arbitrary basis to orthogonal using PCA is required. In accordance with the mathematical model of PCA in the matrix form $\mathbf{X}$ and $\mathbf{Y}$ are represented as

$$
\begin{aligned}
& \mathbf{Y}=\mathbf{L}_{Y} \mathbf{F}_{Y}, \\
& \mathbf{X}=\mathbf{L}_{X} \mathbf{F}_{X}
\end{aligned}
$$

where $\mathbf{Y}, \mathbf{X}$-matrices of centered and normalized parameters, $\mathbf{F}$ - the matrix of principal components, $\mathbf{L}$-the load matrices of principal components.

To find the matrix of principal component loadings $\mathbf{L}$ you need to solve the equation

$$
(\mathbf{R}-\mathbf{\Lambda} \mathbf{I}) \mathbf{U}=0
$$

where $\mathbf{R}=\frac{1}{k-1} \mathbf{Y} \mathbf{Y}^{\mathrm{T}}$-the correlation matrix, $\mathbf{Y}^{\mathrm{T}}$ - the transposed matrix of centered and normalized features, $\boldsymbol{\Lambda}$-the diagonal matrix of eigenvalues, $\mathbf{I}-$ the identity matrix, $\mathbf{U}-$ the matrix of eigenvectors. 
The solution of equation (4) is defined by the condition $\mathbf{U}^{\mathrm{T}} \mathbf{U}=1$. Eigenvectors $\mathbf{U}$ are orthogonal due to the fact that they correspond to different eigenvalues of a symmetric matrix $\mathbf{R}$. Knowing the matrix of eigenvectors $\mathbf{U}$ and eigenvalues $\Lambda$, a matrix of principal component loadings can be found

$$
\mathbf{L}=\mathbf{U} \Lambda^{1 / 2}
$$

In order to apply the PCA the information about the original projection matrices $\mathbf{X}$ and $\mathbf{Y}$ coordinate system with bases $x_{1}, x_{2}, \ldots x_{n}$ и $y_{1}, y_{2}, \ldots y_{n}$.is required. Using that information and the procedure described above we can transit to a new orthogonal coordinate system with bases $U_{X}\left(u_{x 1}, u_{x 2}, \ldots, u_{x n}\right)$ и $U_{Y}\left(u_{y 1}, u_{y 2}, \ldots, u_{y n}\right)$.

Thus, by using PCA, a transition from the original to new matrix into orthogonal projected coordinate system is carried out, which are linked by relation

$$
\mathbf{F}_{Y}=\mathbf{P F}_{X},
$$

where $\mathbf{P}$ - the transformation matrix of principal components.

The main components $\mathbf{F}_{Y}$ and $\mathbf{F}_{X}$ are projections of $\mathbf{Y}$ and $\mathbf{X}$ on the basis of vectors $\mathbf{U}_{Y}$ and $\mathbf{U}_{X}$. It is well known that the coordinate transformation matrix of vector projection in the process of basis change constitutes a transponsed matrix to the inverse matrix of the transition from the old to the new base. If it is known that

$$
\mathbf{U}_{\mathrm{Y}}=\mathbf{S} \mathbf{U}_{\mathrm{X}},
$$

where $\mathbf{S}=\mathbf{U}_{\mathrm{Y}} \mathbf{U}_{\mathrm{X}}^{-1}$ - the transition matrix between the bases $\mathbf{U}_{\mathrm{X}}$ and $\mathbf{U}_{\mathrm{Y}}$, then the transformation matrix of principal components based on orthogonal eigenvectors is

$$
\mathbf{P}=\left(\mathbf{S}^{-1}\right)^{\mathrm{T}}=\mathbf{U}_{Y} \mathbf{U}_{X}^{\mathrm{T}}
$$

Substituting (5) into (2) we can get

$$
Y=L_{Y} \mathbf{P} L_{X}^{-1} X
$$

From the last expression (6), matrix A model has the form

$$
\mathbf{A}=L_{Y} U_{Y} U_{X}^{\mathrm{T}} L_{X}^{-1} .
$$

Thus we obtain the model connecting the two multicollineare values of matrix $\mathbf{X}$ and $\mathbf{Y}$.

Let's consider the example of such model preparation binding the magnetic characteristics of the permanent magnet (PM) before the production process and after. To build a model we need to form a training set (consisting of a magnetic characteristics family) including magnetic parameters $n$ PM having the same history. The information on the PM magnetic characteristics (e.g. demagnetization curve) can be represented in different ways. In statistical modeling vector-matrix the representation of the original data is used. We agree to denote the vector magnetic parameters of the PM prior to the processing of technological operation $X$, and a vector of magnetic parameters of the PM after the processing of technological operation Y.

Thus, if we introduce the notation, each $i$-th sample is represented by a vector $X=\left[x_{1}, x_{2}, \ldots x_{m}\right]$ characterizing its properties before the processing, and the vector $Y=\left[y_{1}, y_{2}, \ldots y_{k}\right]$ describing the properties of the sample after the processing. In general, the number of elements of the vectors $X$ and $Y$ is different, i.e. $m \neq k$

Let us form the matrix of available vectors $\mathbf{X}$ and $\mathbf{Y}$ : 


$$
\mathbf{X}=\left[\begin{array}{cccc}
x_{11} & x_{12} & & x_{1 m} \\
x_{21} & x_{22} & \cdots & x_{2 m} \\
& \vdots & & \\
x_{n 1} & x_{n 2} & \cdots & x_{n m}
\end{array}\right] \quad \mathbf{Y}=\left[\begin{array}{cccc}
y_{11} & y_{12} & & y_{n k} \\
y_{21} & y_{22} & \cdots & y_{2 k} \\
& \vdots & & \\
y_{n 1} & y_{n 2} & \cdots & y_{n k}
\end{array}\right]
$$

Each row in the obtained matrices contains the values of intensity or polar radius of a demagnetization curve. Since the number of the PM included in the learning sample before and after the processing is $\mathrm{n}$, the number of rows in the matrices $\mathbf{X}$ and $\mathbf{Y}$ is equal to $n$. The number of columns in the matrix is determined by the number of points on the demagnetization curve. In our case PM measurements before processing were carried out at $m$ points, and after processing in the $k$ points. Each of the matrix $\mathbf{X}$ columns can be considered as an independent variable - influencing factor, and the lines - as observations on this variable. The same is true for the matrix $\mathbf{Y}$.

However, two processes discussed do not always show multicollinearity. In this case, it is necessary to build multi-physical model using only one raw data matrix with PCA. [5]. In accordance with a mathematical PCA model represented in matrix form let's consider $\mathbf{Y}$ matrix as:

$$
\mathbf{Y}=\mathbf{L}_{Y} \mathbf{F}_{Y}
$$

To find the loads matrix of principal components $\mathbf{L}$ it is necessary to solve the equation (2) as in the previous case.

Thus, considering the transition of matrix $\mathbf{Y}$ into the new basis (7) and substituting it into the equation (1) we will have the form

$$
L_{Y} F_{Y}=A X
$$

from here matrix model $\mathbf{A}$ is

$$
\mathbf{A}=L_{Y} F_{Y} X^{-1}
$$

In this case we obtain the model connecting the matrix $\mathbf{X}$ and multicollinear matrix $\mathbf{Y}$.

The use of this model allows express-diagnostics of the state of proportional electromagnets of hydraulic drives, by measuring and setting in the model only time-current characteristics.

For such express-diagnostics [13-20], you can also apply the elements of the main component method. A software product has been developed that allows one to determine the type of defect of a proportional electromagnet in the form of current and the form of flux linkage.

\section{Conclusion}

Thus, when constructing an adequate model of multiphysical processes for carrying out a field experiment, it is necessary to take into account the phenomenon of multicollience of the initial data obtained as a result of measurements. The most effective method of combating multicollience is the application of the principal component method for the transition to a new orthogonal basis. The experimental studies have shown that by using this approach, you can get an adequate model. 
The results of the work were obtained with the support of the project No. 2.7193.2017/8.9 "Development of the scientific basis for designing, identifying and diagnosing high- precision positioning systems using the inverse electrical engineering methodology", performed within the framework of the main parts of the state task. The results of the work were obtained using the equipment of the collective use center "Diagnostics and Energy-Efficient Electrical Equipment" of the YRSU Platov South-Russian State Polytechnic University (NPI).

\section{References}

1. A.M. Lankin, M.V. Lankin, M.Y. Lankina, IOP Conf. Ser.: MSE, 10, 012074 (2017)

2. N.I. Gorbatenko, M.V. Lankin, Elektrotekhnika, 8, 55 (2004)

3. A. Lankin, D. Shaykhutdinov, V. Grechikhin, D. Schuchkin, N. Narakidze, N. Gorbatenko, Jour. of Eng. and Appl. Sci., 12(2), 363 (2017)

4. B.T. Polyak, M.V. Khlebnikov, Automation and Telemechanics, 3, 130 (2017)

5. V.V. Grechikhin, M.V. Lankin, A.M Lankin, Prom-Eng. WISTC, 235 (2015)

6. N.I. Gorbatenko, A.M. Lankin, M.V. Lankin, Procedia Engineering, 129, 799 (2015)

7. A.M. Lankin, M.V. Lankin, M.Y. Lankina, IOP Conf. Ser.: MSE, 10, 012075 (2017)

8. N.I. Gorbatenko, A.M. Lankin, M.V. Lankin, D.V. Shayhutdinov, Inter. Jour. of Appl. Eng. Res., 10(3), 6509 (2015)

9. A.M. Lankin, M.V.Lankin, N.I. Gorbatenko, D.V. Shayhutdinov, MAS, 9(8), 247 (2015)

10. D.V. Shaikhutdinov, A.M. Lankin, N.D. Narakidze, V.V. Grechikhin, K.M. Shirokov, N.I. Gorbatenko, Res. Jour. of Appl. Sci., 10(10), 555 (2015)

11. A.M. Lankin, M.V. Lankin, V.V. Grechikhin, D.V. Shaikhutdinov, Res. Jour. of Appl. Sci., 10(10), 695 (2015)

12. N.I. Gorbatenko, A.M. Lankin, M.V. Lankin, Procedia Engineering, 150, 1027 (2016)

13. A.M. Lankin, M.V. Lankin, O.A. Naugolnov, Procedia Engineering, 150, 1020 (2016)

14. N.I. Gorbatenko, A.M. Lankin, M.V. Lankin, Met. Sci. and Heat Treat., 1-3 (2017)

15. N.I. Gorbatenko, A.M. Lankin, M.V. Lankin, Met. Sci. and Heat Treat., 4-6 (2017)

16. N.I. Gorbatenko, V.V. Grechikhin, M.V. Lankin, A.M. Lankin, Meas. Tech., 59(7), 747 (2016)

17. A.M. Lankin, M.V. Lankin, Nat. Ass. of Sch., 4-2 (9), 132 (2015)

18. A.M. Lankin, Mod. high tech., 9-3, 420 (2016)

19. A.M. Lankin, Bas. res., 9-3, 495 (2016)

20. M.V. Lankin, A.M. Lankin, IOP Conf. Ser.: MSE, 10, 012031 (2016) 